\title{
Chronic Kidney Disease Management - What Can We Learn from South African and Australian Efforts?
}

\author{
I.J. Katz ${ }^{a}$ W.E. Hoy ${ }^{b}$ S. Kondalsamy-Chennakesavan ${ }^{b}$ T. Gerntholtz ${ }^{a}$ \\ J. Scheppingen $^{\mathrm{b}}$ S. Sharma ${ }^{\mathrm{b}}$ O. Butler ${ }^{\mathrm{a}}$ E. Shezi ${ }^{\mathrm{a}}$ G. Mdleleni ${ }^{\mathrm{a}}$ \\ D. Mthombenic
}

aDumisani Mzamane African Institute of Kidney Disease, University of the Witwatersrand, Soweto, South Africa; 'bidney Disease Research and Prevention (KDRP), and Centre for Chronic Disease, University of the Queensland, Central Clinical School, Royal Brisbane Hospital, Brisbane, Australia; ${ }^{\mathrm{C}}$ Gauteng Health Department, Johannesburg, South Africa

\section{Key Words}

Chronic kidney disease $\cdot$ Chronic disease outreach programs $\cdot$ End-stage renal disease

\begin{abstract}
Background: The prevalence of chronic kidney disease is on the rise. Our objective is to describe two programs to improve the awareness and management of hypertension, renal disease, and diabetes in remote Australian Aboriginal and urban and periurban South African communities. We focus on how the Australian Aboriginal and South African Chronic Disease Outreach Programs have worked together. Methods: The establishment of prevention programs in developing countries is a challenge. The paper evaluates these challenges, including accessing international aid. The programs advocate that regular integrated checks for chronic disease and their risk factors are essential elements of regular adult health care. Programs should be run by primary health workers, following algorithms for testing and treatment, and a backup provided by nurse coordinators. Constant evaluation is essential to develop community health profiles and adapt program structure. Results: Both programs are discussed, including how they are organized to de-
\end{abstract}

liver preventative and treatment strategies. The challenges and adaptations required are outlined. Conclusions: It is the aim of the international kidney community to prevent chronic kidney disease. The South African and Australian groups highlight the need for a systematic and sustained approach to the management of chronic diseases to achieve this goal.

Copyright $@ 2006$ S. Karger AG, Basel

\section{Introduction}

There is a surge in the rise of chronic diseases such as diabetes and hypertension worldwide. By 2020, the burden of diabetes and cardiovascular disease will have increased by $130 \%$ in Africa alone, with concomitant increases in prevalence of chronic kidney disease (CKD) and end-stage renal disease (ESRD) [1]. A similar alarming situation exists among the Australian Aboriginal communities. An epidemic of renal and related chronic diseases has appeared in remote Australian Aboriginal communities since the early 1980s. Death rates are greatly elevated [2,3], whereas incidence rates of treated ESRD in remote areas are up to 1,500 per million, and in some individual communities $\geq 3,000$ per million $[4,5]$. Ne-

\section{KARGER}

Fax +4161306 1234 E-Mail karger@karger.ch www.karger.com 
phrologists and governments have realized that the costs of providing dialysis are prohibitive and that the answer to this problem may be prevention. The burden of CKD is heaviest and most costly in poorer communities, where resources for dialysis are scarce to non-existent. Thus, in these countries, money for dialysis should be used for prevention and primary care; however, the challenges are great.

It is against this backdrop that early detection and treatment of CKD is so attractive. The International Society of Nephrology highlights the epidemic of CKD and explains that the markers and risk factors are known [6]; therefore, learning the practical ways of establishing prevention programs is an essential tool for the nephrologist.

\section{Chronic Disease Outreach Programs - Establishment and Methods}

\section{International Aid for the Development of Health Programs and Research}

Although international aid organizations express wonderful ideals to provide help for needy communities, they are not always possible to achieve. There is no substitute for formal health structures. Although international aid organization groups like the World Health Organization, Médecins Sans Frontières, Initiative for Cardiovascular Health in Developing Countries and the Global Forum for Health Research aim to complement formal health sectors, there remains an ongoing moral dilemma in providing health care to vulnerable populations [7], including a developing dependence on external assistance which minimizes the responsibility of a government to deliver health care. Additionally, the projects can be manipulated by existing governments or mask the real problems [8]. Nephrologists can learn from the experiences of these organizations when attempting to establish prevention programs.

Nephrologists have a few options to consider before starting a program. Firstly, a CKD prevention program can be established on its own with the primary focus on the kidney as the organ to treat. Secondly, CKD can fall under the gambit of all chronic illnesses, recognizing that the management of the key chronic illness risk factors such as hypertension and diabetes will have the greatest impact on reducing the progression of kidney disease [7]. The broad range of kidney problems and their etiologies makes the nephrologist well suited to follow an integrated approach, as is suggested by the WHO [9].

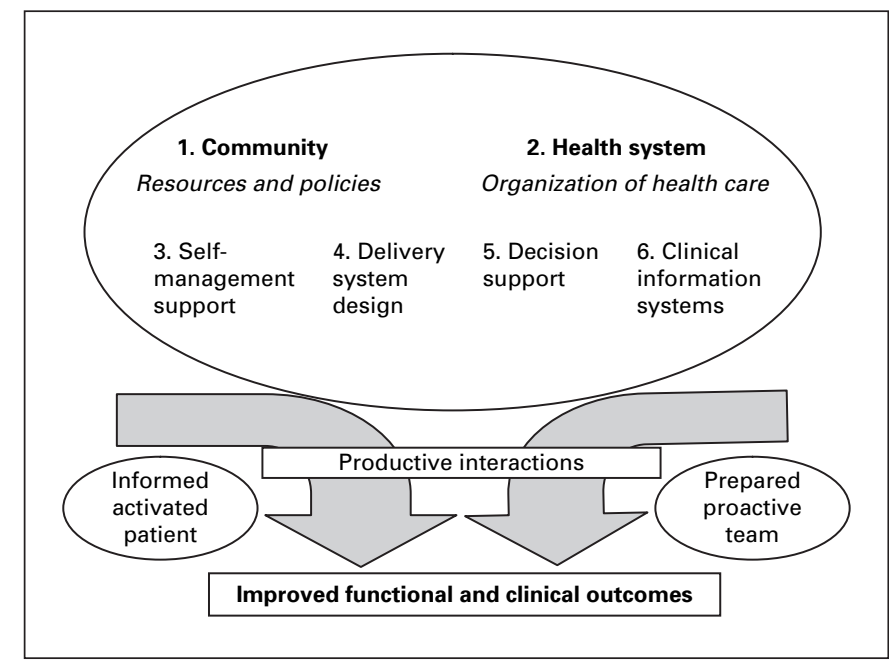

Fig. 1. Wagner Chronic Illness Care Model. This model was used to design phase II of the SACDOP.

\section{Sharing Efforts to Establish Prevention Programs}

Wendy Hoy and her team in Australia are probably the most experienced in establishing kidney and cardiovascular protection programs. They have helped develop programs in Australian Aboriginal communities, as well as in other countries including India, Nigeria and South Africa (SA). The key to this development is to engage community interest in chronic illness management and prevention strategies, to help assess needs in the community and to develop an agreement with the local community or health authority [10]. Once these basic requirements have been met, one can go on to help the local staff implement the program and strive to ensure sustainability by providing the mechanisms or skills to evaluate processes and outcomes. In 1996, Ivor Katz met Wendy Hoy at an African nephrology meeting where they realized that problems were similar and there was potential to utilize the experiences of the Australians to develop an SA program. Phase I (2001-2003) of the SA program ran in two regions with limited staff using adapted Australian protocols. Government regional managers collected the data sheets, and monthly report meetings took place with all primary health care clinicians. Phase II (2004-2005) started after a training visit to Australia, and then, 2 Australian nurse coordinators were relocated to train 2 local nurses in SA. Protocols were rationalized and adapted based on the experiences of phase I. The Wagner Chronic Illness Care Model was used as the template to fashion phase II (fig. 1) [11]. The 2 SA nurse coordinators and the program director visit clinics weekly to collect data, pro- 
vide feedback, support decisions and run focus groups. Every 4 months, meetings are held with primary health care clinicians and regional health managers to look at trends in the data and provide focused education. Phase II was only run in region $\mathrm{A}$, the reasons being both practical and due to staff limitations recognized during the organization of phase I.

\section{The Australian Outreach Program}

The Australian outreach program ran under the banner of KDRP, Kidney Disease Research and Prevention, but has since changed its name to Chronic Disease Outreach Program (ACDOP). This name change emphasizes the change in the program focus from its beginnings on the Tiwi Islands with the main emphasis on kidney disease to what it is now, an integrated focus on chronic disease. The outreach program evolved from the initial program in a remote Australian Aboriginal community (Tiwi Islands). This program showed marked reduced sickness, hospital admissions, ESRD, and natural deaths [12, 13]. The Australian outreach program remains an ideal template on which to develop a prevention program $[10,14]$. It contains both screening and treatment components and has since been extended to other Aboriginal areas in Australia. It involves mass community screening with the aim of detecting high-risk groups and providing treatment. The communities range from a few hundred to a couple of thousand.

The program offers services to reduce the harmful impact of chronic 'lifestyle' diseases, particularly diabetes, high blood pressure and kidney disease. The Kidney Disease Research and Prevention Program focuses on the identification and modification of risk factors, early diagnosis of chronic disease and systematic treatment to modify its course.

Australia offered to teach and model these same concepts and practices for the SA communities so they can be incorporated and subsequently sustained in their ongoing adult health care plans. This service was offered for free, without any conditions, and funding was provided from the local government, research funds and from the Australian outreach program.

\section{SA Chronic Disease Outreach Primary Prevention Program}

Arising from the Australian experiences, the SA experience is similar, but also unique. The elements of the program are summarized in table 1. In SA, the database is paper based and data are loaded at one center. Interactive review, interactive dialogue and multi-party consul-
Table 1. Principles of the CDOP for remote Aboriginal communities and urban and periurban SA communities

Regular testing of all adults for chronic disease and risk factors and treatment for those in need are essential components of adult health care

The program is run by primary health care clinicians who follow algorithms for testing and treatment

Use of a limited number of medicines to maximize familiarity and to save costs

Nurse coordinators provide backup, from a distance, so that one serves several communities/regions

Doctors provide backup for problematic cases and treatment questions, so that one can serve an entire region

Maximum use of telephone, fax, e-mail and web for communications

Computerized database in Australia and in development in SA

Constant evaluation of individual profiles, community profiles, processes and outcomes

Table 2. Regular checkup: CDOP minimal elements

- Chart review: existing diagnoses, complications, parity, contraception and medicines

- History: smoking, drinking, drugs, existing diagnoses, menstruation

- Weight, height (once)

- Blood pressure

- Skin examination for sores, scabies, fungal infections in some communities

- Urine dipstick for protein, blood, nitrites, leukocytes

- Capillary fingerstick for random glucose

- Some common additional tests according to algorithms

- Repeat urine if apparently contaminated

- Urine culture if apparently infected

- Urine ACR if dipstick 1+, if diabetic, hypertensive or history of proteinuria

- Fasting glucose if random glucose suspicious

- $\mathrm{HbA}_{1 \mathrm{c}}$ if diabetic

- Full blood count if anemic

- Serum creatinine if overt albuminuria (ACR 34+)

- Electrolytes if considering ACEI treatment

- Lipids, sometimes

- Involve the participant

- Feedback and education

- Start treatment or alter treatment on first/baseline visit if needed/possible

$\mathrm{ACR}=$ Albumin creatinine ratio; $\mathrm{HbA}_{1 \mathrm{c}}=$ hemoglobin $\mathrm{A}_{\mathrm{cc}}$.

tation occur through regular meetings and visits to the participating health centers. Patient information and the laboratory data are collected at the local clinic and then evaluated by the nurse coordinators. The components of 


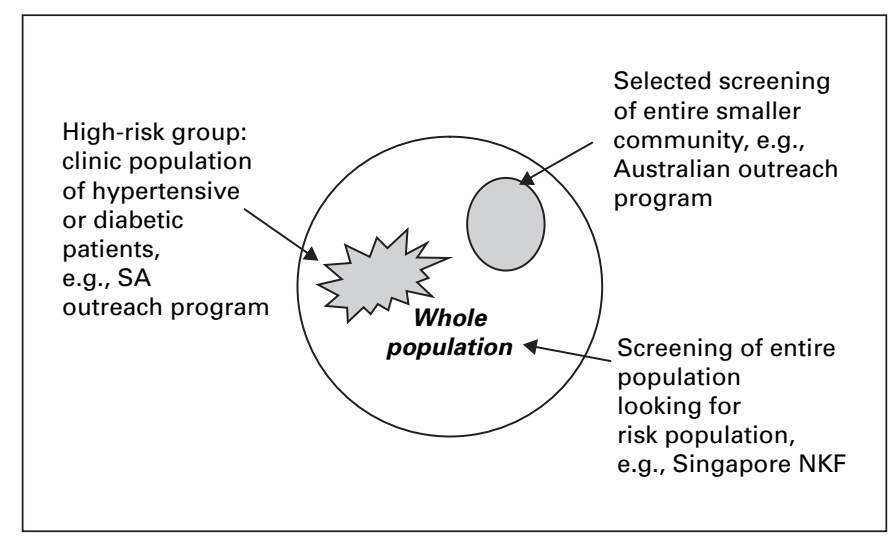

Fig. 2. Diagram indicating the different approaches and strategies for prevention programs for CKD depending on resources and skills. $\mathrm{NKF}=$ National Kidney Foundation.

testing are shown in table 2 . Reports are drawn up outlining suggested patient management and follow-up plans (fig. 1, decision support). Follow-up data are then collected every 4 months, or earlier if required. High-risk patients (uncontrolled hypertension, diabetes and proteinuria or proteinuria alone) are screened and evaluated for the program by the local primary health care nurses at the local clinic. A different screening policy occurred in SA due to the much higher patient population and staff limitations (fig. 2).

When patients are found to have problems which cannot be managed at the primary care clinics, these patients are referred to a tertiary hospital for ongoing management. These problems include a glomerular filtration rate $<60 \mathrm{ml} / \mathrm{min} / \mathrm{m}^{2}$, i.e. CKD stages 3 or 4 , patients with CKD stage 5 (ESRD) who require dialysis, patients who require an HMGCoA reductase inhibitor for cholesterol control, and possibly uncontrolled diabetes and hypertension for $>8$ months at the primary care clinic. In both programs, there is an emphasis on starting angiotensinconverting enzyme inhibitors (ACEI) early.

A brief examination of heart and lungs and peripheral pulses is included in the evaluation. Some of the emphases reflect local problems (e.g., skin infections/infestations) and might not be important in other settings. Other elements can be added to optimize the use of a regular encounter, such as immunizations, sexually transmitted diseases and HIV checks.

The SA program takes place in Soweto, reflecting a people in transition from a healthy traditional to a westernized lifestyle, as is the case for Aboriginal communities. This transition has brought people at a high risk for chronic diseases such as diabetes, hypertension and kidney disease. Against a known background of poor blood pressure and glucose control, the program was implemented in 20 primary care clinics in Soweto and nearby regional clinics [15]. A tertiary hospital serves as the focal point for data collection and program management, as well as for patient referral. The program is an integrated chronic illness program focusing on detecting high-risk patients and controlling their hypertension, diabetes and risk factors $[11,16]$. The program is developed around an integrated chronic illness model which includes simplifying the targets for blood pressure, glucose and proteinuria control, providing ongoing education and support with patient management as well as regularly evaluating the achievement of set clinical targets. The program also has a qualitative component which focuses on the primary health care team and includes focus group interviews and diary records of the nurse coordinators.

\section{Findings}

\section{The ACDOP}

The program has significantly reduced morbidity and mortality in patients with kidney and cardiovascular disease $[13,14]$. It has influenced national protocols, becoming a government lobby group and galvanizing non-government organizations in Australia [14].

Forty-one percent of women and $72 \%$ of men were smokers. Most men were current users of alcohol, but most women were not. The mean body mass index (BMI) varied from community to community making it impossible to infer weight in all Aboriginal people based on results from one community. Rates of hypertension, proteinuria and diabetes were strongly correlated with increasing age in all communities, as demonstrated for a single community in figure 3 . There are a large number of young and middle-aged adults with morbidities. Average rates of these morbidities, although excessive in all, differed at least 2-fold among communities, as shown in figure 4. Proteinuria and hypertension were early features of the health profile, whereas diabetes was a later and more variable element (fig. 3). Coexistence of multiple morbidities was common in middle-aged and inevitable in older people. Figure 5 shows such integration in one community, which had intermediate rates of morbidities. Morbidities were also strongly correlated with measures of body weight, including measurement of the waist, and with birth weight (inversely, and for women only). Women had more renal disease and diabetes than men, where- 


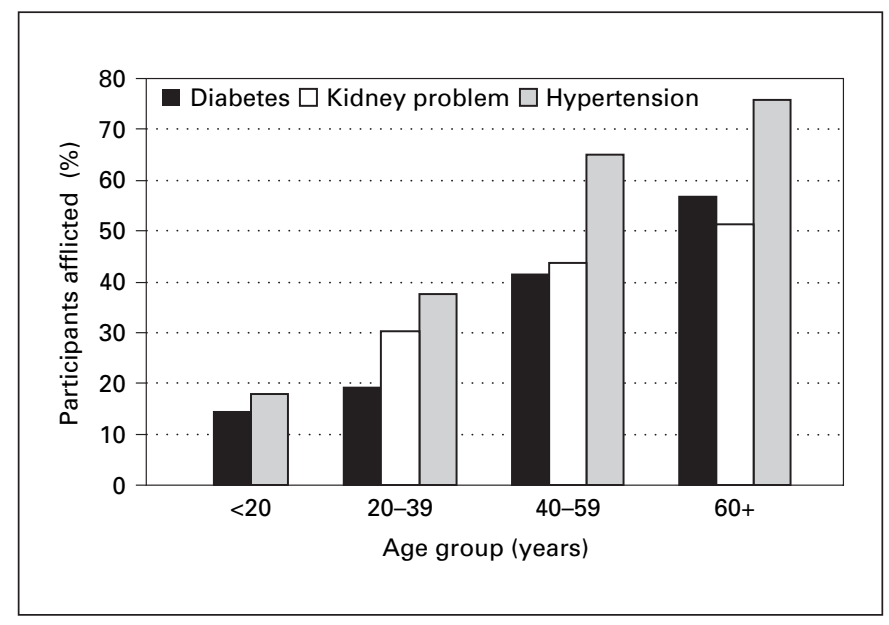

Fig. 3. Prevalence of chronic conditions by age group in a single Aboriginal community participating in the CDOP. The rates increase with age, which may suggest something about the pathophysiology of chronic disease in the population. This pattern provides the case for repeated testing throughout adult life.

as men had higher rates of hypertension. Alcohol use was associated with an almost doubled rate of hypertension in women.

\section{The SA Chronic Disease Outreach Primary \\ Prevention Program}

The development of the SA program is an example of contact with an international aid organization. The program was developed with the support of Australia and adapted to SA conditions. The first phase contained a surveillance component, providing baseline data; it achieved success with blood pressure control, but had only limited success with diabetes control and proteinuria reduction. Against a known background of poor blood pressure and glucose control, phase I was implemented in 16 primary care clinics in Soweto and nearby regional clinics. Eight hundred and seventy-one patients were screened ( $67 \%$ females, $33 \%$ males). Ages ranged from 20 to 70 years, most patients being between 51 and 60 years old. Twelve percent of participants smoked, and $14.5 \%$ admitted to drinking alcohol. There was a significant difference in age, risk factors and chronic diseases between the different regions screened (table 3). Almost 60\% of all subjects screened were obese according to BMI and waist measurements (fig. 6), and in phase II $(n=530)$, this continues to be a major problem with 59\% of patients being obese. Risk factors may differ by region, as shown in the Australian Aboriginal communities. Thirty-five percent

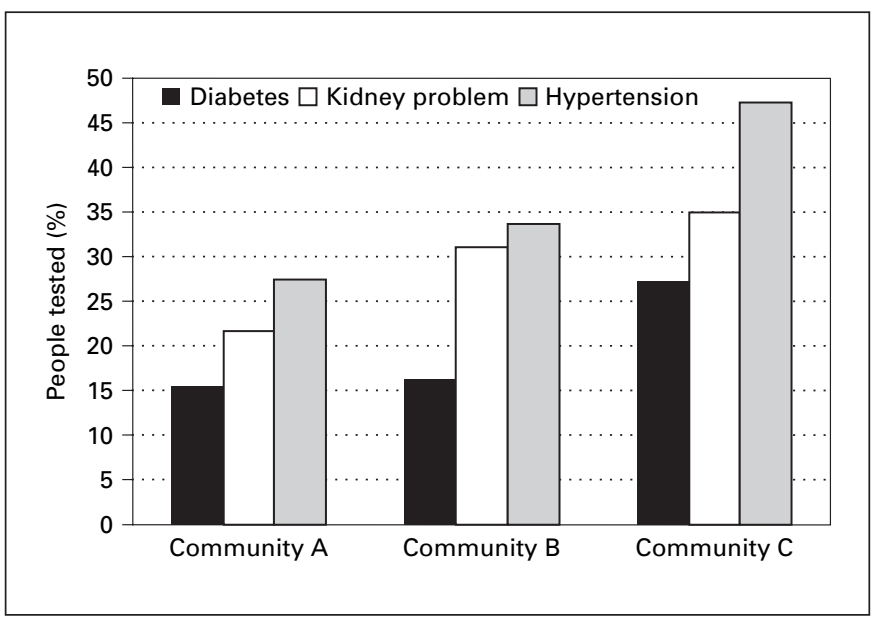

Fig. 4. Estimated rates of chronic disease morbidities in communities participating in the ACDOP. The rates of morbidities differ by community, which suggest that pilot studies are needed to anticipate required services.

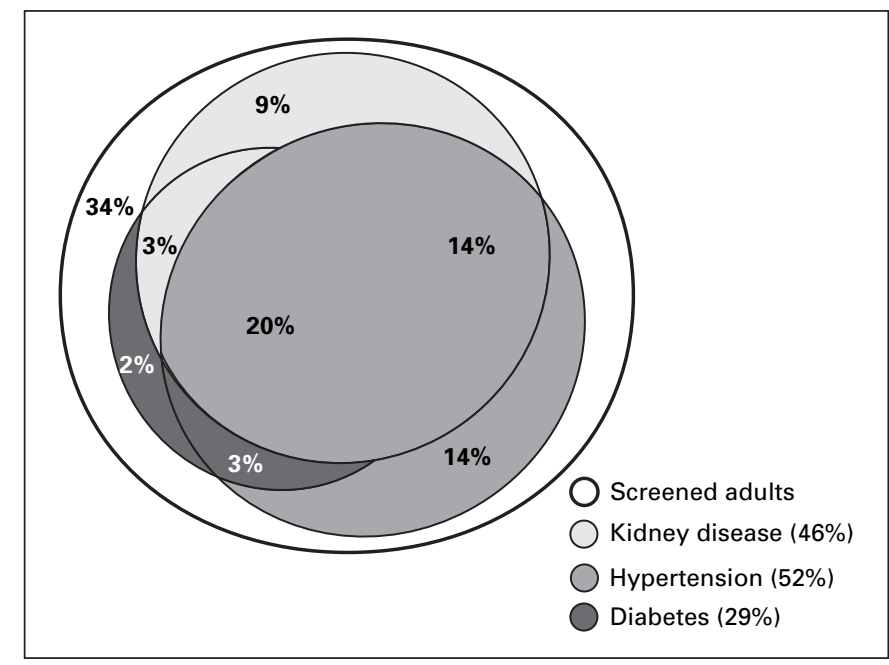

Fig. 5. Overlapping morbidities of chronic disease among screened adults in a single Aboriginal community in the CDOP.

of patients had evidence of CKD in the form of proteinuria. There was a significant reduction in blood pressure in all active participants in phase I (fig. 7), and similar results have occurred in phase II over a 20-month followup. ACEI treatment was initiated or uptitrated in those patients with indications for ACEI; $82 \%$ of patients with diabetes and $98 \%$ of diabetics with albuminuria had been prescribed ACEI. Blood pressure values also showed sig- 


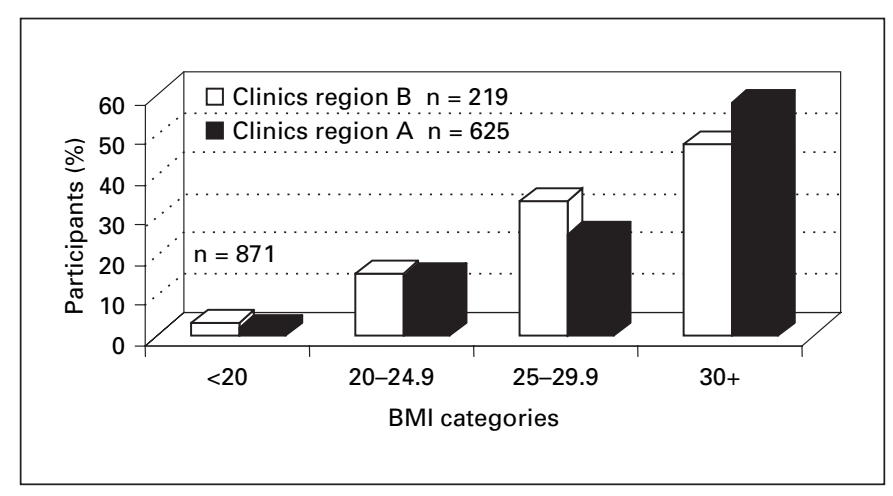

Fig. 6. Distribution of BMI among participants in the pilot phase I of the SACDOP between the clinics of two regions.

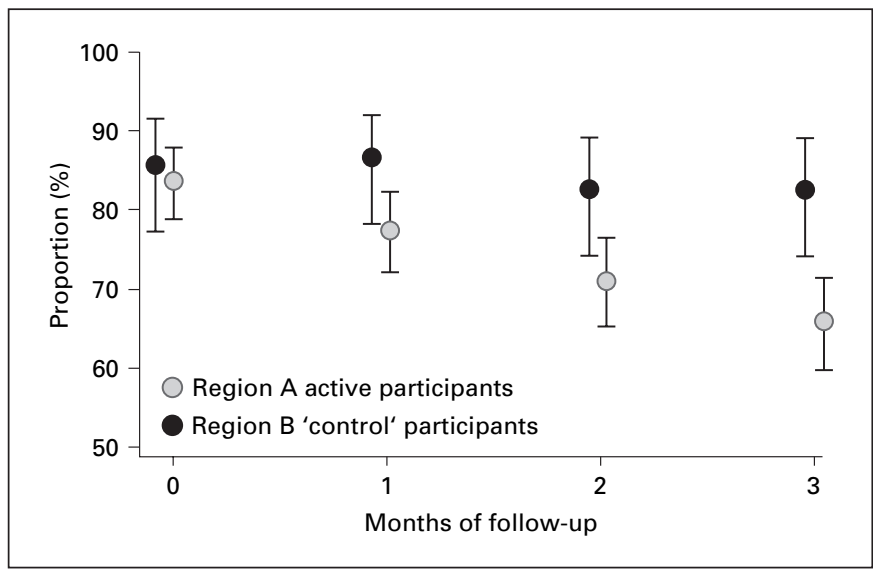

Fig. 7. Changes in the proportion of CDOP participants with uncontrolled blood pressure in the pilot phase I clinics by region. Bars indicate confidence intervals.
Table 3. Baseline profile of patients in the SACDOP phase I pilot program in clinics of two different regions

\begin{tabular}{llcllrr}
\hline Parameters & \multicolumn{2}{l}{ Region A clinics } & & \multicolumn{2}{l}{ Region B clinics } & p value \\
\cline { 2 - 3 } & $\mathrm{n}$ & & $\mathrm{n}$ & & \\
\hline Age, years & 219 & $51.4 \pm 10.0$ & & 625 & $57.5 \pm 11.9$ & $<0.001$ \\
Diabetics & 226 & $110(48.7)$ & & 645 & $234(36.3)$ & 0.001 \\
Hypertensive & 226 & $178(78.8)$ & & 645 & $622(96.4)$ & $<0.001$ \\
Smokers & 201 & $19(9.5)$ & & 577 & $86(14.9)$ & 0.051 \\
BMI & 162 & $31.0 \pm 8.3$ & & 581 & $32.7 \pm 8.6$ & 0.026 \\
SBP, mm Hg & 221 & $147.8 \pm 22.2$ & & 619 & $153.0 \pm 21.6$ & 0.002 \\
\hline
\end{tabular}

Data are means \pm SD or proportions given in parentheses. All parameters show significant difference between the two regions. SBP $=$ Systolic blood pressure. nificant improvement in those patients starting ACEI and in those with hypertension at baseline.

Diabetes control was particularly poor in phase I, but participants did show an improvement in control. Average blood glucose was $>12 \mathrm{mmol} / \mathrm{l}$ at baseline, and at 6 months, nearly $75 \%$ had random non-fasting blood glucose levels $<12 \mathrm{mmol} / \mathrm{l}$. In phase II, with more aggressive use of insulin, there was better diabetes control, showing mean random serum glucose $\leq 8 \mathrm{mmol} / 1$ in $81 \%$ and hemoglobin $A_{1 c}<8$ in $73 \%$ of patients. A reduction in proteinuria from 0.057 to $0.050 \mathrm{~g} / \mathrm{mmol}$ occurred, but was not significant. Hyperlipidemia was common, with a mean level of $5.2 \mathrm{mmol} / \mathrm{l}$ in a traditionally low-risk population. A number of weaknesses in phase I are outlined below.

Most importantly, of all the subjects screened, at least $10 \%$ were found to have significant problems which re- quired referral, and most had stage 3 or greater CKD (fig. 8).

\section{Program Activities}

The workload gradually defined itself in the following categories: (1) regular screening, which can be predicted and scheduled, according to the population base in the Australian Aboriginal communities and according to poor control in a high-risk patient population in SA; (2) follow-up of subjects with equivocal or abnormal results, which can happen on the same encounter, or on other additional encounters, depending on the disease burden; (3) initiation, upward titration and monitoring of treatment, followed by a multiplication effect, with the total workload depending on the burden of disease, the complexity of an individual's problems and his or her response to intervention; therefore, in some Australian communities, 


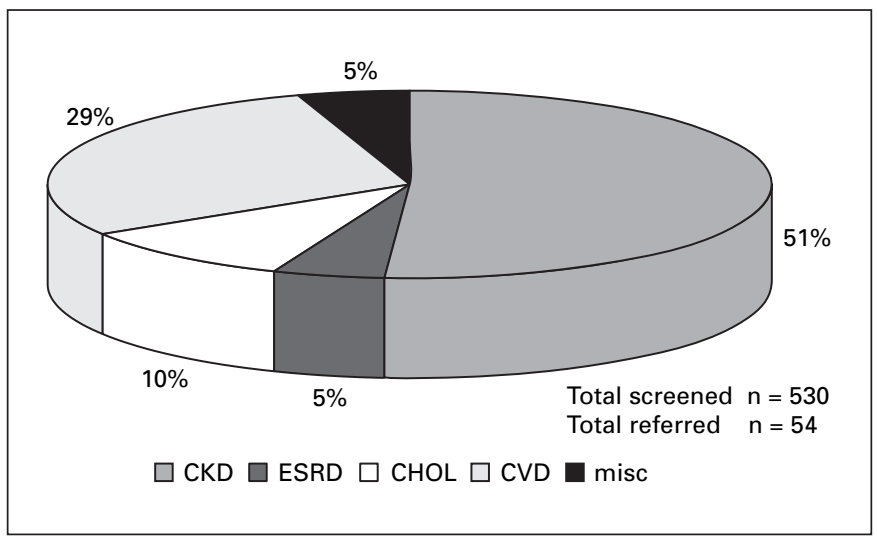

Fig. 8. Clinical indications for referral to a tertiary hospital for further management or evaluation. $\mathrm{CHOL}=$ Serum cholesterol level; CVD = cardiovascular disease (heart failure, ischemia, angina); misc $=$ miscellaneous reasons .

this was impossible to achieve, and in SA, it relied on the involvement of voluntary primary health care nurses; (4) catch up, recording and restocking; (5) clinic and staff meetings; (6) community and individual education, and (7) education and training updates.

\section{Staffing and Follow-Up Problems}

The fundamental difficultly in the programs was staff shortage of local health workers and frequent absenteeism in Australia, and attrition in SA clinics. These issues have a great impact on health care. The causes are complex and deep-seated, but improved incentives for health workers, proper pay scales, empowerment, respect and good backup are needed. High drop-up rates, up to $62 \%$ in both phases of the SA program, have occurred due to staffing shortages, the inability of the nurses to fill in follow-up data when patients return, as well as cutting of costs by the local regional health service. Continuity of care is a major problem in SA with clinics seeing 3,000 5,000 chronic disease patients per month and patient nurse ratios of $1: 50$ or 1:60. There are only 2 nurse coordinators for the follow-up of patients. Follow-up was thus dependent on local clinic support. All primary health care nurses were reluctant to be involved and nurses involved were not always available due to days off and having to rotate to other areas within the clinic (i.e. acute patient care).

Ongoing challenges in SA include the lack of coordinator personnel at each site to facilitate the daily management of the program. While communication linkages improved access to doctors at the tertiary care facility, de- mands on time and the public health care system 'culture' remained a barrier.

\section{Discussion and Conclusions}

Improvement in glucose levels due to a focus on glucose control in phase II of the SACDOP occurred as a result of the poor control found in phase I. There was a focus on early insulin use in poorly controlled patients. The benefits of improved diabetes control was not seen in the Australian program [14]. Despite education and focus groups on weight reduction, very little has been achieved in this area in SA. These examples reflect the dynamic nature of running a prevention program and the need for a systematic, sustained and flexible approach.

In SA, it is important to realize that the $10 \%$ of patients referred to the tertiary hospital with severe disease burden, most CKD 3 or greater, would not otherwise have been detected. This is not to mention the improvement in control of some of the risk factors in the general patient population participating in the program, e.g., blood pressure and diabetes control. These detections lead to additional cost for the governments; however, they have to realize that the long-term benefits are cost-effective [17].

It is difficult to compare outcomes in the different programs because the focuses of the programs are slightly different and findings indicate that results are not always transferable between communities or regions. The Australians noted that generalizations about risk factors and resultant chronic conditions could not be made for all Aboriginal people as the dominant chronic disease in the metabolic syndrome varied between communities [14]. It was noted that morbidities are best considered elements of a single vascular/renal/metabolic syndrome, rather than separate entities. In SA, hypertension and diabetes are the dominant metabolic syndrome burden. From the results and discussion, it can be seen that appropriate changes in focus can be made according to ones findings. Populations, communities and regions may be different, and this must be taken into account when establishing prevention programs.

Despite shortages of staff and problems with running the programs, the improvements in risk reduction, if sustained, should result in a reduction in morbidity and mortality.

There is a raised awareness for an integrated approach to the management of chronic conditions. The WHO and initially Wagner have popularized similar models of integrated care $[9,18]$. These models capture the complex- 
ity of providing health care for chronic conditions in an organized way [9]. Hopefully, non-governmental chronic disease organizations like heart foundations and kidney foundations will work together more closely in tackling common risk factors. More resources have to be found to support the key players in this type of integrated approach, especially for primary health care clinicians. The Australian Government has undertaken to supply the Ferret computerized information technology health care system to the communities or services it supports, although more attention is needed for startup and ongoing training when such systems are used [14, 19]. Computerization is possibly even more important in developing countries where these health systems need to improve efficiency, especially with the higher patient loads and lower doctor/nurse patient ratios. In SA, collaboration with a Canadian-based medical data bank company and a local IT company will hopefully help computerize the program.

Community profiles, like those described here, provide much information for planning needs-based health services [15]. Disease rates may change with age and community which impacts in the health delivery for different communities. Early detection and prevention programs inform appropriate use of screening tests and treatment resources.

It should be noted that the coexistence of morbidities highlights the deficiencies and inefficiencies of specialtyspecific models and programs.

A CKD prevention program is a simple and effective means of ensuring improved management of CKD and cardiovascular disease. The close Australian and SA working relationship has provided positive outcomes and a model for international aid, as well as better understanding of chronic diseases in their communities. Development of international networks is critical to prevent CKD and especially ESRD.

One has to focus not only on the technical and scientific aspects, but also on supporting or caring for the staff, the paradox being that continuing care delivered by a well-functioning team is the basis on which the control of chronic disease must rest [20].

\section{Acknowledgements}

Professor Helen Schneider (School of Public Health, University of the Witwatersrand) and Professor Sarala Naicker (Department of Nephrology, University of the Witwatersrand).

\section{References}

1 Schena FP: Epidemiology of end-stage renal disease: international comparisons of renal replacement therapy. Kidney Int 2000;57:3945.

2 Statistics ABo: The health and welfare of Australia's Aboriginal and Torres Strait Islander people, 2003. ABS catalogue No 4704.0, August 2003. Canberra, Australian Bureau of Statistics, 2005.

3 Dempsey KE, Condon JR: Mortality in the Northern Territory, 1979-1997. Darwin, Territory Health Services, 1999.

-4 Spencer JL, Silva DT, Snelling P, Hoy WE: An epidemic of renal failure among Australian Aborigines. Med J Aust 1998;168:537-541.

5 McDonald SP, Russ GR: Burden of end-stage renal disease among indigenous peoples in Australia and New Zealand. Kidney Int 2003; 63:S123-S127.

6 Crouser W: ISN to lead global fight against kidney disease. ISN News, Aug 2005, pp 1-15.

7 Katz I: International aid and medical practice in the less-developed world: doing it right, what can renal organizations learn? Kidney Int Suppl 2005;68:S60-S65.

8 Einterz EM: International aid and medical practice in the less-developed world: doing it right. Lancet 2001;357:1524-1525.
Epping-Jordan JE: Integrated approaches to prevention and control of chronic conditions. Kidney Int Suppl 2005;98:S86-S88.

10 Hoy WE, Scheppingen J, McKendry K, Sharma S, Kondalsamy-Chennakesavan S: Planning services for noncommunicable chronic disease (NCDs) in Aboriginal communities (abstract). International Society of Nephrology European Dialysis and Transplant Association-European Renal Association World Congress of Nephrology, Berlin, 2003.

11 Wagner EH, Davis C: Improving Chronic Illness Care (ICIC). Seattle, The Robert Wood Johnson Foundation, 2004.

-12 Hoy WE, Baker PR, Kelly AM, Wang Z: Reducing premature death and renal failure in Australian Aboriginals. A community-based cardiovascular and renal protective program. Med J Aust 2001;174:201-202.

13 Hoy WE, Wang Z, Baker PR, Kelly AM: Reduction in natural death and renal failure from a systematic screening and treatment program in an Australian Aboriginal community. Kidney Int 2003;63:S66-S73.

14 Hoy WE, Kondalsamy-Chennakesavan S, Scheppingen-Smith J, Sharma S, Katz I: A chronic disease outreach program for Aboriginal communities. Kidney Int 2005;68:S76S82.
15 Mohammed DES: Gauteng Health Department report on hypertension and diabetes control at Soweto clinics. Johannesburg, Gauteng Health Department, June 2000.

16 Katz I, Luyckx V, Butler O, Hopley M: An early evaluation of the Primary Prevention Program (PPP), a kidney disease renoprotection program (KDRP) in Soweto, South Africa. South African Renal Society Biannual Congress, Bloemfontein, 2002.

17 Baker PRA, Hoy W, Wang Z, Glaziou P: Preventing renal failure in Australian Aborigines: an effectiveness and cost-analysis of a screening and treatment program. 35th Annu Sci Meet Aust NZ Soc Nephrol, Brisbane, 1999.

18 Wagner E: Meeting the needs of chronically ill people. BMJ 2001;323:945-946.

19 Hoy WE, Kondalsamy-Chennakesavan S: Final report on the Aboriginal Chronic Disease Outreach Program to the Office of Aboriginal and Torres Strait Islander Health and Kidney Health Australia. Brisbane, University of Queensland, October 2004.

20 Epping-Jordan J: The challenge of chronic conditions: WHO responds. BMJ 2001;323:947948 . 